\title{
Higgs decay into four charged leptons in presence of dimension six operators
}

\author{
Ambresh Shivaji* \\ Centre for Cosmology, Particle Physics and Phenomenology (CP3), \\ Université Catholique de Louvain, B-1348 Louvain-la-Neuve, Belgium \\ E-mail: ambresh.shivaji@uclouvain.be
}

\begin{abstract}
We study the effects of dimension six operators on the Higgs decay into four lepton channel. The calculation of new matrix element is performed in the Higgs basis and it is implemented in a Monte Carlo event generator. The code also allows the calculation in other popular choices of basis for the dimension six operators. We have considered all the relevant operators, both the CPeven and $\mathrm{CP}$-odd operators, which contribute to this decay channel. Choosing some benchmark values for the parameters of the Higgs basis, we compare our predictions for partial decay width and some important kinematic distributions with the corresponding NLO(EW) SM predictions.
\end{abstract}

25th International Workshop on Deep Inelastic Scattering and Related Topics 3-7 April 2017

University of Birmingham, Birmingham, UK

\footnotetext{
*In collaborations with S. Boselli, C. M. Carloni Calame, G. Montagna, O. Nicrosini, F. Piccinini [1]
} 


\section{Introduction}

In absence of any hint of new resonances at the LHC, it is important that we measure the properties of the Higgs boson more precisely, specially its couplings with fermions and gauge bosons. In this context, the model independent parameterizations of new physics (NP) appear more appealing than the model dependent ones. The simplest of these is the $\kappa$-framework in which the Higgs boson couplings with gauge bosons are scaled by a common factor $\kappa_{v}$ and its coupling with fermions by factor $\kappa_{f}$. The current experimental bounds allow a deviation of 10-20\% in these parameters from their standard model $(\mathrm{SM})$ values $\left(\kappa_{v}^{\mathrm{SM}}=\kappa_{f}^{\mathrm{SM}}=1\right)$ [2]. Although simple, the $\kappa$-framework is not a gauge invariant parametrization of new physics beyond $\mathrm{LO}$, and it does not capture the kinematic effects due to NP at the LO. Generalizations of $\kappa$-framework in terms of anomalous vertices/phenomenological Lagrangian, pseudo observables and Effective Field Theory (EFT)-framework are well discussed in the literature [3].

In an EFT framework, the effect of new physics beyond the SM (BSM) can be captured by higher dimension operators constructed using the SM degrees of freedom and consistent with the SM gauge symmetry. A set of gauge invariant independent operators of a given mass dimension form a basis. Unlike $\kappa$-framework, EFT gives rise to new structure to SM vertices and also introduces new LO vertices. Some popular choices of bases include Warsaw, SILH and Higgs $[4,5,6,7]$. The choice of a basis is usually led by the convenience of minimizing the number of parameters required to capture the BSM effects on a given class of observables.

Although the $H \rightarrow 4 \ell$ decay channel has low event rates, it has a very high signal-overbackground ratio. It is mainly sensitive in gluon fusion production channel. The combined signal strength measured at the LHC is $\mu_{g g \mathrm{~F}, 4 \ell}^{\mathrm{CMS}+\mathrm{ATLAS}}=1.13_{-0.31}^{+0.34}$ [8]. Together with $H \rightarrow \gamma \gamma$, it has a high mass resolution, $m_{H}^{\text {CMS }+ \text { ATLAS }}=125.09 \pm 0.24 \mathrm{GeV}$. Due to its non-trivial kinematics, the $H \rightarrow 4 \ell$ decay channel is suitable for probing new physics effects $[9,10,11,12,13,14,15]$.

In my talk I will advertise an updated version of the Hto 41 code which is used to study NP effects in $H \rightarrow 4 \ell$ decay channel within an EFT framework. The code is available for public use.

\section{2. $H \rightarrow 4 l$ in Higgs basis}

The Higgs basis [7] is designed to parameterize new physics effects in the Higgs sector. The interaction Lagrangian is constructed using the mass eigenstates. The parameters of the Higgs basis are connected to the Wilson coefficients of dimension-six operators in a given basis via linear transformations. The number of independent parameters of the Higgs basis are same as the number of independent dimension-six operators. We consider the parts of the Higgs basis Lagrangian relevant to $H \rightarrow 4 \ell$ decay. The anomalous $H V V(V=\gamma, Z)$ coupling is parameterized by five CPeven and three CP-odd parameters,

$$
\begin{aligned}
\mathscr{L}_{D=6}^{H V V} & =\frac{H}{v}\left[\left(1+\delta c_{Z}\right) \frac{1}{4}\left(g_{1}^{2}+g_{2}^{2}\right) v^{2} Z_{\mu} Z^{\mu}+c_{\gamma \gamma} \frac{e^{2}}{4} A_{\mu v} A^{\mu v}+c_{Z \gamma} \frac{e \sqrt{g_{1}^{2}+g_{2}^{2}}}{2} Z_{\mu v} A^{\mu v}\right. \\
& +c_{Z Z} \frac{g_{1}^{2}+g_{2}^{2}}{4} Z_{\mu v} Z^{\mu v}++c_{Z \square} g_{2}^{2} Z_{\mu} \partial_{v} Z^{\mu v}+c_{\gamma \square} g_{1} g_{2} Z_{\mu} \partial_{v} A^{\mu v}+ \\
& \left.+\tilde{c}_{\gamma \gamma} \frac{e^{2}}{4} A_{\mu v} \tilde{A}^{\mu v}+\tilde{c}_{Z \gamma} \frac{e \sqrt{g_{1}^{2}+g_{2}^{2}}}{2} Z_{\mu v} \tilde{A}^{\mu v}+\tilde{c}_{Z Z} \frac{g_{1}^{2}+g_{2}^{2}}{4} Z_{\mu v} \tilde{Z}^{\mu v}\right] .
\end{aligned}
$$


In the above, the parameter, $c_{\gamma}$ is not independent

$$
c_{\gamma \square}=\frac{1}{g_{2}^{2}-g_{1}^{2}}\left[2 g_{2}^{2} c_{Z \square}+\left(g_{2}^{2}+g_{1}^{2}\right) c_{Z Z}-e^{2} c_{\gamma \gamma}-\left(g_{2}^{2}-g_{1}^{2}\right) c_{Z \gamma}\right] .
$$

The anomalous $Z \ell \ell$ and $H Z \ell \ell$ couplings are parameterized by,

$$
\begin{gathered}
\mathscr{L}_{D=6}^{Z \ell \ell}=\sqrt{g_{1}^{2}+g_{2}^{2}} \sum_{\ell=e, \mu} Z_{\mu}\left[\bar{\ell}_{L} \gamma^{\mu} I_{W, \ell}^{3}-s_{W}^{2} Q_{\ell}+\delta g_{L}^{Z \ell \ell} \ell_{L}+\bar{\ell}_{R} \gamma^{\mu}-s_{W}^{2} Q_{\ell}+\delta g_{R}^{Z \ell \ell} \ell_{R}\right], \\
\mathscr{L}_{D=6}^{H Z \ell \ell}=2 \frac{\sqrt{g_{1}^{2}+g_{2}^{2}}}{v} \sum_{\ell=e, \mu}\left[\delta g_{L}^{H Z \ell \ell} H Z_{\mu} \bar{\ell}_{L} \gamma^{\mu} \ell_{L}+\delta g_{R}^{H Z \ell \ell} H Z_{\mu} \bar{\ell}_{R} \gamma^{\mu} \ell_{R}\right] .
\end{gathered}
$$

In the Higgs basis, the $H V \ell \ell$ couplings are not independent, and $\delta g_{L}^{Z \ell \ell}=\delta g_{L}^{H Z \ell \ell}, \delta g_{R}^{Z \ell \ell}=\delta g_{R}^{H Z \ell \ell}$.

The publicly available Hto $4 \mathrm{I}$ code, can provide precise predictions for Higgs decay into four charged leptons with NLOEW+PS accuracy [16]. The calculation of new matrix elements for $H \rightarrow 2 e 2 \mu$ and $H \rightarrow 4 e / 4 \mu$ is carried out in FORM [17], and it is implemented in a new version of Hto 41 code. The matrix elements are given in Higgs basis. To allow the user a flexibility in the choice of basis, an independent dictionary between the parameters of the Higgs basis and the Wilson coefficients of the EFT bases like SILH and Warsaw is also implemented ${ }^{1}$. In the code, $\delta g_{i}^{Z \ell \ell}$ and $\delta g_{i}^{H Z \ell \ell}$ are kept independent. The code has a provision to include or drop the quadratic dependence on the parameters.

\section{Numerical results}

We first discuss the modification in the partial decay width due to the presence of the Higgs basis parameters. For $H \rightarrow 2 e 2 \mu$, the ratio of width in BSM and SM is given by,

$$
\frac{\Gamma_{\mathrm{BSM}}}{\Gamma_{\mathrm{SM}}}(H \rightarrow 2 e 2 \mu)=1.00+\sum_{i} X_{i} c_{i}+\sum_{i j} X_{i j} c_{i} c_{j}+\sum_{i j} \tilde{X}_{i j} \tilde{c}_{i} \tilde{c}_{j}
$$

where $c_{i}=\left\{\delta c_{Z}, c_{\gamma \gamma}, c_{Z \gamma}, c_{Z Z}, c_{Z \square}, \delta g_{L}^{Z \ell \ell}, \delta g_{R}^{Z \ell \ell}, \delta g_{L}^{H Z \ell \ell}, \delta g_{R}^{H Z \ell \ell}\right\}$ and $\tilde{c}_{i}=\left\{\tilde{c}_{\gamma \gamma}, \tilde{c}_{Z \gamma}, \tilde{c}_{Z Z}\right\}$. Since the partial decay width is a CP-even observable, the terms linear in CP-odd parameters do not appear. The coefficients of linear $\left(X_{i}\right)$ and quadratic $\left(X_{i j}\right.$ and $\left.\tilde{X}_{i j}\right)$ terms are sensitive to the SM input parameters and selection cuts applied on the final state leptons. For $M_{H}=125 \mathrm{GeV}$ and a minimum cut of $15 \mathrm{GeV}$ on the invariant mass of the same flavour opposite sign lepton pairs we get,

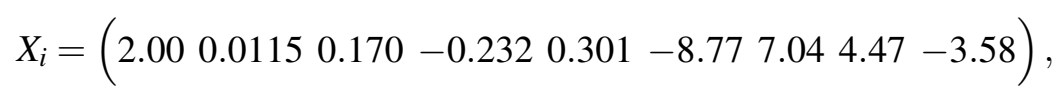

\footnotetext{
${ }^{1}$ See the appendix B in [1].
} 


$$
\begin{aligned}
X_{i j}=\left(\begin{array}{ccccccccc}
1.00 & 0.0115 & 0.170 & -0.232 & 0.301 & -8.77 & 7.04 & 4.47 & -3.58 \\
0 & 0.055 & 0.0706 & -0.0312 & -0.0448 & -0.227 & -0.179 & -0.181 & 0.174 \\
0 & 0 & 0.768 & -0.490 & -0.702 & -3.47 & -2.80 & 2.81 & 2.740 \\
0 & 0 & 0 & 0.114 & 0.273 & 2.23 & 0.696 & -1.55 & -0.873 \\
0 & 0 & 0 & 0 & 0.265 & 0.566 & 3.41 & -0.974 & -2.51 \\
0 & 0 & 0 & 0 & 0 & 25.4 & -15.4 & -25.9 & 7.85 \\
0 & 0 & 0 & 0 & 0 & 0 & 22.0 & 7.85 & -22.4 \\
0 & 0 & 0 & 0 & 0 & 0 & 0 & 7.85 & -1.58 \\
0 & 0 & 0 & 0 & 0 & 0 & 0 & 0 & 7.50
\end{array}\right) \\
\\
\tilde{X}_{i j}=\left(\begin{array}{ccccc}
0.0487 & -0.00745 & 0.0000910 \\
0 & 0.308 & -0.00592 \\
0 & 0 & 0.00317
\end{array}\right) .
\end{aligned}
$$

The relative importance of individual parameters in modifying the partial decay width is governed by the size of these coefficients. For example, among CP-even parameters related to gaugeHiggs coupling, the effect of $c_{Z \gamma}$ is the largest. The modification in width due to $c_{Z \gamma}$ is dominated by the coefficient of quadratic term. The effect of $c_{Z \square}$ is larger than $c_{Z Z}$ and opposite in nature. This is due to the fact that the linear terms $\left(X_{Z \square}, X_{Z Z}\right)$ have opposite signs and are more relevant than the corresponding quadratic terms. Like $c_{Z \gamma}$, the CP-odd parameter $\tilde{c}_{Z \gamma}$ affects width the most. The parameter $\tilde{c}_{Z Z}$ has almost no effect on the width due to a very small value of the corresponding coefficient $\left(\tilde{X}_{i j}\right)$. Note that some of these couplings like $\delta g_{i}^{Z \ell \ell}, c_{\gamma \gamma}$ and $\tilde{c}_{\gamma \gamma}$ are already well constrained by the available data from LEP and LHC, respectively.

Next we study the effect of the BSM parameters on kinematic distributions which can help in discriminating various parameters. For the sake of simplicity and to emphasize the role of distributions, we consider the values of the parameters which are consistent with a $30 \%$ deviation in partial decay width. We consider only those parameters which are less constrained by the data. We find that in the Higgs rest frame the angular variables, $\Delta \theta_{e^{-}} \mu^{-}$(the angle between $e^{-}$and $\mu^{-}$) and $\phi$ (angle between the decay planes of the intermediate gauge bosons) are most sensitive to the BSM parameters.

In Fig. 1, we compare the BSM predictions due to $c_{Z Z}$ and $c_{Z \square}$ with the corresponding SM predictions at $\mathrm{LO}$ and NLO+PS. We find that the effect of two parameters on angular observables is opposite in nature. Numerically, the effect can be as large as $45 \%$ in certain bins. The solid and dashed lines in these plots refer to the calculation done with or without including the quadratic term. The effect of quadratic term is more visible in $c_{Z \square}$.

In Fig. 2, we display angular distributions for CP-odd parameter $\tilde{c}_{Z \gamma}= \pm 1$ and compare them with the corresponding predictions in the SM. Both these values are roughly consistent with $30 \%$ enhancement in partial decay width. As we would expect, the angular distributions, in particular, the angle $\phi$ is sensitive to the linear term in $\tilde{c}_{Z \gamma}$ and also to its sign.

\section{Summary and outlook}

We have developed a new version of Hto 4 l event generator, which allows the study of $H \rightarrow$ 


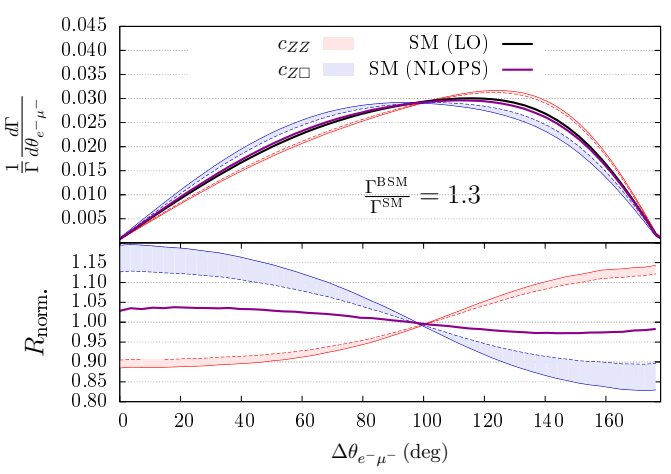

(a)

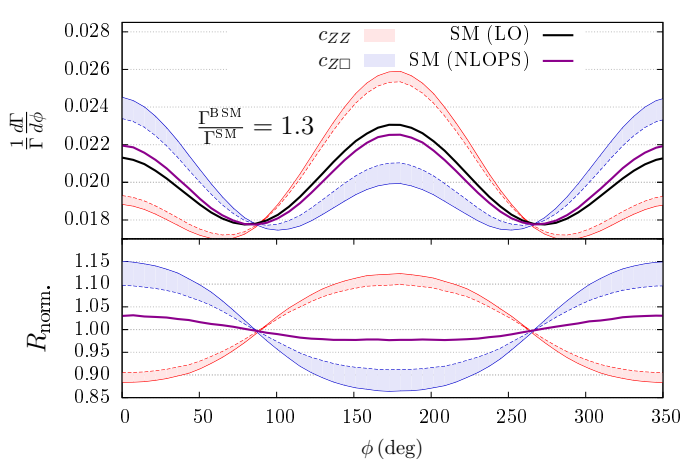

(b)

Figure 1: Kinematic distributions for CP-even parameters. Upper panel: normalized distribution for angular variables $\Delta \theta_{e^{-}} \mu^{-}$(left) and $\phi$ (right). Lower panel: Ratio of normalized distribution in BSM and in SM.

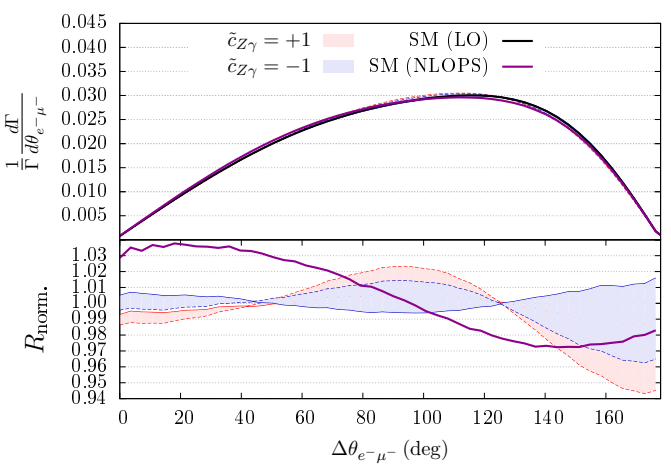

(a)

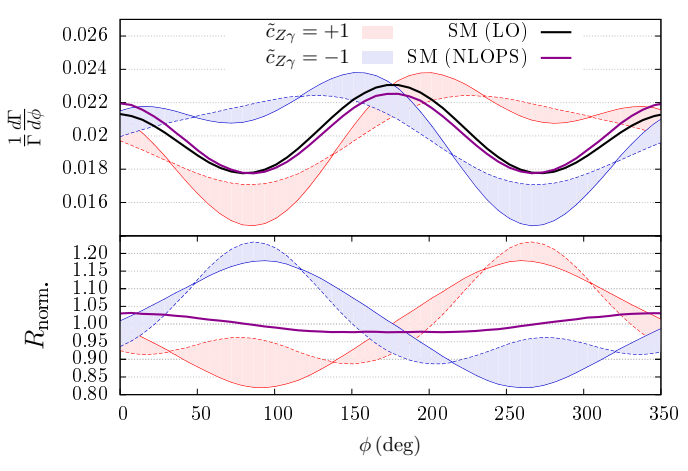

(b)

Figure 2: Kinematic distributions for CP-odd parameter. Upper panel: normalized distribution for angular variables $\Delta \theta_{e^{-}} \mu^{-}$(left) and $\phi$ (right). Lower panel: Ratio of normalized distribution in BSM and in SM.

$2 e 2 \mu$ and $H \rightarrow 4 e / 4 \mu$ decay in presence of dimension-six operators. The BSM matrix elements can be calculated independently in the Higgs, Warsaw and SILH bases. In our simplified study, we find that the angular observables like $\phi$ and $\Delta \theta_{e^{-}} \mu^{-}$can discriminate among various parameters of the dimension-six operators. In near future we plan to update the code with the implementation of $H \rightarrow 2 l 2 v$ BSM matrix elements and, include the parton shower effects on BSM predictions.

\section{Acknowledgments}

AS would like to thank the organizers of DIS-2017 and the conveners of the WG3. He would like to acknowledge the financial support from MOVE-IN Louvain Cofund grant and the IISN "Fundamental interaction" convention 4.4517.08.

\section{References}

[1] S. Boselli, C. M. Carloni Calame, G. Montagna, O. Nicrosini, F. Piccinini and A. Shivaji, arXiv:1703.06667 [hep-ph]. 
[2] G. Aad et al. [ATLAS and CMS Collaborations], Measurements of the Higgs boson production and decay rates and constraints on its couplings from a combined ATLAS and CMS analysis of the LHC pp collision data at $\sqrt{s}=7$ and $8 \mathrm{TeV}$ JHEP 1608, 045 (2016) [arXiv: 1606.02266 [hep-ex] ].

[3] C. Mariotti and G. Passarino, Int. J. Mod. Phys. A 32, no. 04, 1730003 (2017) doi:10.1142/S0217751X17300034 [arXiv:1612.00269 [hep-ph]].

[4] G. F. Giudice, C. Grojean, A. Pomarol and R. Rattazzi, JHEP 0706, 045 (2007) doi:10.1088/1126-6708/2007/06/045 [hep-ph/0703164].

[5] B. Grzadkowski, M. Iskrzynski, M. Misiak and J. Rosiek, JHEP 1010, 085 (2010) doi:10.1007/JHEP10(2010)085 [arXiv:1008.4884 [hep-ph]].

[6] R. Contino, M. Ghezzi, C. Grojean, M. Muhlleitner and M. Spira, JHEP 1307, 035 (2013) doi:10.1007/JHEP07(2013)035 [arXiv:1303.3876 [hep-ph]].

[7] D. de Florian et al. [LHC Higgs Cross Section Working Group], doi:10.23731/CYRM-2017-002 arXiv:1610.07922 [hep-ph].

[8] https://atlas.web.cern.ch/Atlas/GROUPS/PHYSICS/CONFNOTES/ATLAS-CONF-2016-079/

[9] D. Stolarski and R. Vega-Morales, Phys. Rev. D 86, 117504 (2012) doi:10.1103/PhysRevD.86.117504 [arXiv:1208.4840 [hep-ph]].

[10] Y. Chen, N. Tran and R. Vega-Morales, JHEP 1301, 182 (2013) doi:10.1007/JHEP01(2013)182 [arXiv:1211.1959 [hep-ph]].77

[11] Y. Chen and R. Vega-Morales, JHEP 1404, 057 (2014) doi:10.1007/JHEP04(2014)057 [arXiv:1310.2893 [hep-ph]].

[12] M. Gonzalez-Alonso, A. Greljo, G. Isidori and D. Marzocca, Eur. Phys. J. C 75, 128 (2015) doi:10.1140/epjc/s10052-015-3345-5 [arXiv:1412.6038 [hep-ph]].

[13] Y. Chen, E. Di Marco, J. Lykken, M. Spiropulu, R. Vega-Morales and S. Xie, JHEP 1501, 125 (2015) doi:10.1007/JHEP01(2015)125 [arXiv:1401.2077 [hep-ex]].

[14] M. Gonzalez-Alonso, A. Greljo, G. Isidori and D. Marzocca, Eur. Phys. J. C 75, 341 (2015) doi:10.1140/epjc/s10052-015-3555-x [arXiv:1504.04018 [hep-ph]].

[15] M. Bordone, A. Greljo, G. Isidori, D. Marzocca and A. Pattori, Eur. Phys. J. C 75, no. 8, 385 (2015) doi:10.1140/epjc/s10052-015-3611-6 [arXiv:1507.02555 [hep-ph]].

[16] S. Boselli, C. M. Carloni Calame, G. Montagna, O. Nicrosini and F. Piccinini, JHEP 1506, 023 (2015) doi:10.1007/JHEP06(2015)023 [arXiv:1503.07394 [hep-ph]].

[17] J. A. M. Vermaseren, math-ph/0010025. 